\title{
Analysis of HMPAO SPECT scans in head injury using Statistical Parametric Mapping
}

\author{
E.A. Stamatakis ${ }^{\mathrm{a}, *}$, J.T.L. Wilson ${ }^{\mathrm{a}}$ and \\ D.J. Wyper ${ }^{\mathrm{b}}$

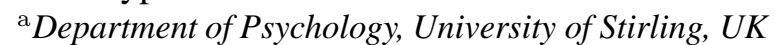 \\ ${ }^{\mathrm{b}}$ Department of Clinical Physics, University of \\ Glasgow, UK
}

\begin{abstract}
The paper examines the ability of Statistical Parametric Mapping (SPM) to contribute towards the quantitative analysis of HMPAO SPECT images containing lesions. A validation study is described in which SPECT images were created that contained synthetic lesions and were analysed with SPM. The study established a set of guidelines concerning the alignment, smoothing, and statistical analysis of images. These were then applied to analysis of SPECT scans from head injured patients. A demonstration is given of the use of SPM to identify localised blood flow abnormalities associated with cognitive deficits after head injury. Correlations between blood flow abnormalities and a test of visual memory are illustrated.
\end{abstract}

\section{Introduction}

SPECT is used to evaluate blood flow abnormalities in a variety of psychiatric and neurological disorders such as dementia and brain trauma. Most HMPAO SPECT studies have employed semiquantitative methodologies or visual inspection for the analysis of the images.

The region of interest (ROI) approach has been used extensively in analysing SPECT images. In Alzheimer's Disease [AD] a number of studies have employed the cortical ring principle [17,19,28,34], which is an elliptical ROI that includes only the cortex. The issue of normalisation to a brain area unaffected by the condition has also been discussed. The most commonly used area is the cerebellum [3,7,23,34]. A

\footnotetext{
*Corresponding author: E. A. Stamatakis, Department of Psychology, University of Stirling, Stirling FK9 4LA, UK. Tel.: +44 01786 467669; Fax: +4401786 467641; E-mail: eas1@ @tir.ac.uk.
}

more sophisticated approach in the context of $\mathrm{AD}$ is that of Houston et al. $[12,13]$. They constructed a normal brain atlas by extracting a mean image from 53 normal controls while images representing correlated normal deviants were identified using principal component analysis. These images formed the "building blocks" of the atlas. Following this, a nearest-normal equivalent was constructed for each subsequent image using the atlas, and was compared to a residual standard deviation image to determine the significance of deviations.

In head injury much of the interpretation has involved blind reading by experienced practitioners. This can be a visual grading scheme based on colour scales $[4,15,16,24,25]$, visual assignment of scores [5, $11,15]$, or classification into categories such as nonfocal, meningial or focal lesions [1], and in other cases diffuse or focal lesions [26,30]. ROIs are by far the most popular method of quantitative analysis of SPECT images in head injury. In some occasions ROIs are drawn manually $[6,14,31]$ and in others anatomically derived templates are used [10].

The manual placement of ROI templates on images is time consuming, and if the images are not aligned to each other or to a standard space, the brain regions measured with the same ROI template could be significantly different. Alignment to a standard space is an issue that receives little attention in SPECT studies. Some groups ensure the immobilisation and positioning of subjects and do not align the images further after they have obtained them. A procedure often used is that of manual alignment to the orbito-meatal line $[6$, $7,11,15,19]$ or a plane near this $[4,25]$. However, this process is subjective.

The use of standard ROI templates can reduce the spatial resolution of the study. A small lesion in a large ROI will produce a minor change in the overall result and is not region specific since the precise location of the lesion will be lost. Conversely, a small ROI applied to a large lesion will not reveal the full extent of the lesion. Comparison of equivalent areas in left and right hemispheres by manually drawing ROIs around 
lesions is also time consuming, subjective, can suffer from localisation problems, and can result in erroneous results if diaschisis is present.

Most of the methods described play an important role in the absence of quantitative methods but when quantification becomes available results will improve and operator subjectivity and low reproducibility will diminish. An automatic method would reduce the time needed to produce results. The use of a universally accepted effective method would allow homogeneity in reported results and inter scientific group comparisons where studies are of a similar nature.

With this objective in mind we have been studying the use of Statistical Parametric Mapping (SPM) [8] as a tool for the identification of lesions. SPM has been used for a considerable time and is becoming accepted as a standard approach in the analysis of brain activation studies. It was designed for PET and MRI images but gradually more research groups are using it to locate perfusion deficits in HMPAO SPECT images [18,20, 35].

We investigated the use of SPM to identify blood flow abnormalities and to estimate their volume. We describe validation studies using simulated lesions, and the application of SPM to data from head injured patients. Finally, we describe the use of SPM to assess correlation between neuropsychological test scores and blood flow deficits resulting from head injury.

\section{Validation of SPM in lesion studies}

\subsection{Tools}

Initially the ability of SPM to identify perfusion deficits was verified with synthetic lesions introduced on a normal image [33]. This image was the average of 32 control spatially normalised images. The lesions were produced by constructing binary masks that contained one lesion each. The corresponding volume was computed on the normal image and the mean intensity was reduced in percentile steps of 10 (down to $-100 \%$ ) for that volume. The lesioned images were constructed on NIH Image 1.61 (developed at the US National Institutes of Health and available at http://rsb.info.nih.gov/nih-image/) on a Power Macintosh 4400/160. SPM was developed at the Wellcome Functional Imaging Laboratory and is available from http://www.fil.ion.bpmf.ac.uk/spm/.

\subsection{Pre-processing}

\subsubsection{Alignment}

There are a number of steps involved in the identification of lesions with SPM. Initially the images are transformed to Talairach space [8]. A choice of linear (up to 12 parameter affine) or non-linear (warping with a linear combination of basis functions) registration is available from SPM.

The ability of SPM'96 to correctly normalise images to standard Talairach space was examined with the set of artificial lesions discussed above. The performance of linear affine transformations was assessed with minimum mean square cost functions. Briefly, the process involved adjusting pitch, roll and yaw (utilising the SPM'96 display function), for 10 spatially normalised images and re-normalising them to a SPECT template. The most acceptable results were produced with 12 parameter linear affine transformation. 12 parameter affine transformation allows optimum matching between object and template images with linear deformation using translations, rotations, zooms and shear. This approach corrects for gross morphological differences across subjects. Non-linear alignment (warping) is used to correct for global variability in head shape. It has been suggested that non-linear image warping can cause erroneous deformation of the images and remove lesions entirely, where gross distortions of brain anatomy are present [2]. A study to examine the usefulness of warping was carried out and it was concluded that when realigning SPECT images that contain lesions the use of 12 parameter linear affine alignment is the best choice in order to avoid compromising anatomical integrity [32]. Given the intrinsic lower spatial resolution of SPECT linear deformations are appropriate.

\subsubsection{Smoothing}

Following spatial normalisation the images are smoothed with an isotropic Gaussian filter to improve the signal-to-noise ratio and reduce errors due to interindividual variation in gyral and sulcal anatomy. The size of the smoothing filter must be selected with the spatial (anatomical) extent of the effect under study in mind. Undersmoothing will increase image noise and the likelihood that spurious single-voxel values survive multiple comparison correction. Oversmoothing may reduce the anatomical specificity making interpretation difficult and may also reduce the statistical significance of the result. The size of filter used in the current studies is $12 \mathrm{~mm}$ FWHM (full width half maximum). 


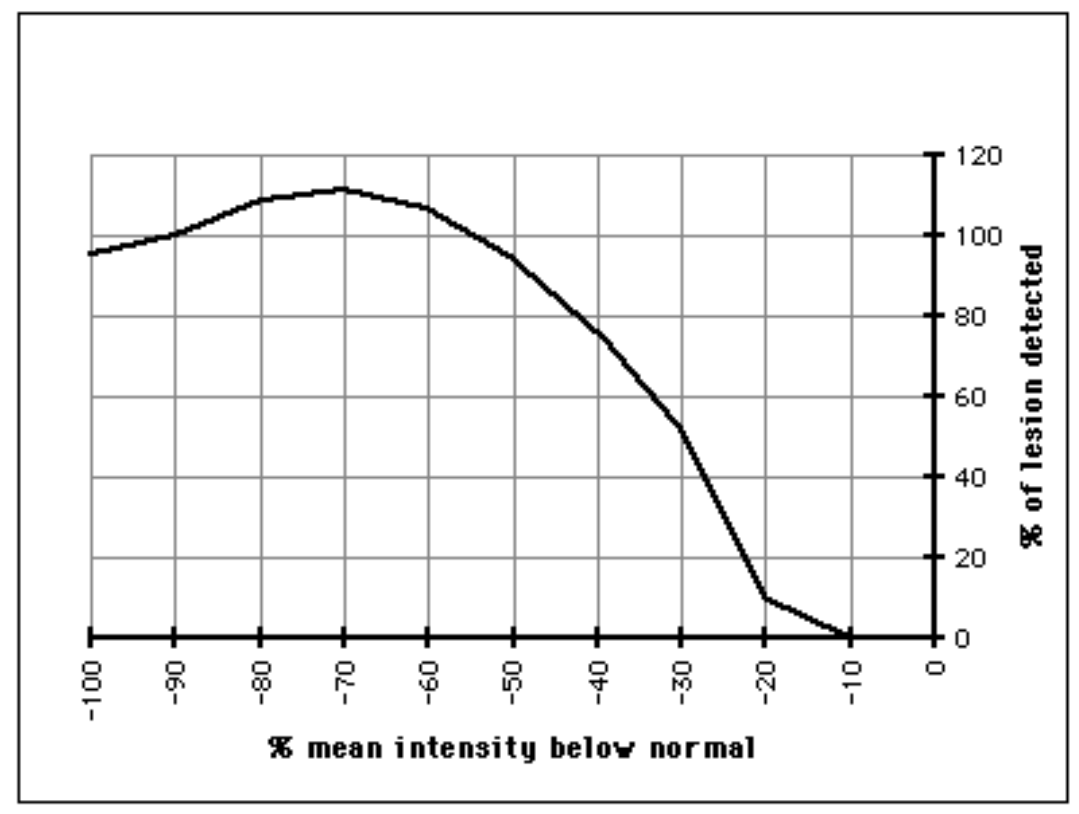

Fig. 1. Percentage of lesion volume (actual volume 32cc) detected by SPM at different mean intensities below normal.

\subsection{Statistical analysis}

For the statistical analysis the appropriate linear regression model has to be chosen. The type of global normalisation, whether or not grand mean scaling is required, and the grey matter threshold must also be selected. Global normalisation and grand mean scaling are used to account for blood flow variations and variation in tracer uptake between individuals. Grey-matter threshold is particularly important in analysing SPECT results from head injured patients because of the morphology of lesions. They are usually regions of low intensity and a choice of a high grey-matter threshold could exclude such areas from the analysis. The height threshold (u) and the corrected p-value, spatial extent threshold $(\mathrm{k})$ are also required and need to be specified. These thresholds denote the significance levels at which results are obtained by SPM and can be modified by the user [9].

Lesion identification was achieved by testing against the 32 controls in SPM'96 with a replication of conditions design. A compare groups: 1 scan per subject design could have also been used since the two types of analysis are statistically equivalent but the first was favoured because of its ease of use. Age was used as a confounding covariate since we found a significant effect when we examined age as a covariate of interest on SPM'96.
The experiment determined the error in volume estimation as a function of lesion depth (percentage reduction from normal) and extent. For example SPM'96 identified an artificial lesion of 32cc when its depth was $-20 \%$ or more. Optimum detection was achieved for lesions at $-50 \%$ from the mean intensity. Grey matter thresholds of 0.2 and 0.5 outperformed that of 0.8. Proportional scaling was found to be more appropriate than an ANCOVA model in the study when used to account for global differences in rCBF in the normalisation stage of SPM. Proportional scaling was used and the grey-matter threshold was set to 0.5 . The results were obtained at $p<0.01$ (with no correction for multiple comparisons) [33]. Figure 1 shows esti mates of a lesion volume for a lesion of $32 \mathrm{cc}$. Smoothing is thought to be responsible for the result exceeding $100 \%$ (overestimating the lesion size) between $-55 \%$ and $-90 \%$. The estimate of the volume decreases after this point since a great number of pixels are left out of the analysis due to their low intensity. The activity is at least as low as that of background pixels and as such they are removed by the grey matter threshold. Detection is quite poor in the region of $0-30 \%$ below normal. It should be possible to quantify this effect and apply a correction factor to the final result by studying the effect of smoothing further and also studying the morphology of real SPECT lesions. The same experiment was carried out for a variety of lesion sizes and it was concluded that with suitable adjust- 

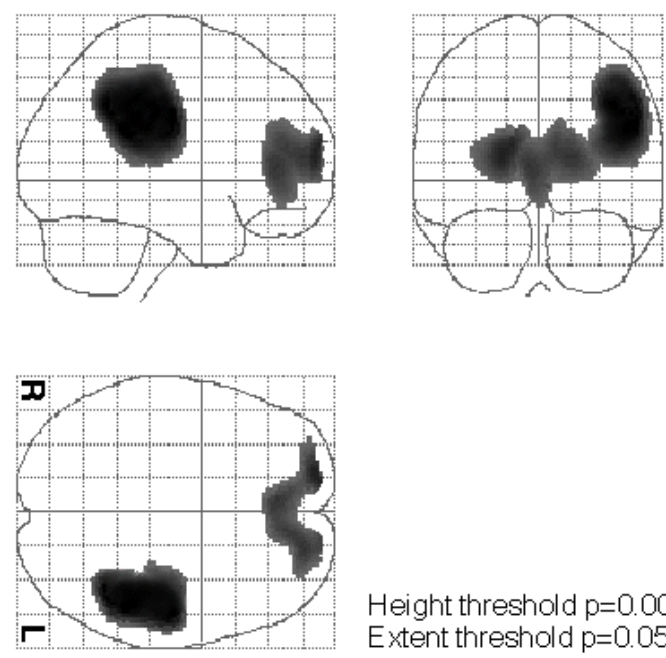

Height threshold $p=0.001$

Extent threshold $p=0.05$ corrected

Fig. 2. Example of blood flow abnormalities detected by SPM in a head injured patient. Maxima for the clusters shown here are at $-42,-37$, $33(Z=6.92)$ in the left parietal lobe and $2,37,6(Z=5.66)$ in the anterior cingulate.

ments of thresholds SPM' 96 is able to assess the size of any SPECT lesion in the range tested and is therefore a useful tool in SPECT lesion studies [33]. A threshold of $p<0.001$ uncorrected was also tested and found to underestimate the artificial lesion volume. Our experience with real lesions (in real images which are more noisy) dictates the use of more conservative thresholds such as $p<0.001$ uncorrected where there exists an a priori hypothesis or $p<0.05$ corrected for multiple comparisons in all other cases.

\section{Application of SPM to head injury}

\subsection{Identification of blood flow abnormalities after head injury}

The experiments with simulated lesions establish SPM's ability to identify abnormalities on HMPAO SPECT images. Following validation, SPM'96 was used in the analysis of scans originating from head injured patients. A total of 103 head injured patients admitted to a specialist neurosurgical unit were recruited to a study that involved acute SPECT and MR and follow-up at 6 months. Of these patients 62 had SPECT and MRI scans that were suitable for this study. The remaining patients were excluded from analysis because they either had acute or follow-up scans only or had incomplete scans. The age of patients ranged from 18 to 60 at the time of injury (age mean $=27.66, \mathrm{SD}=$
10.07). Patients had no prior history of head injury (leading to loss of consciousness), intracranial operation, psychiatric illness treated by hospitalisation, treatment for alcoholism or drug abuse, epilepsy, or mental handicap. On first admission to hospital 28 patients had Glasgow Coma Scale (GCS) scores of 13-15, 9 had GCS scores of 9-12, and 25 had GCS scores of 3-8. MRI images were analysed in a semi-automated ROI fashion using software provided by the scanner's manufacturer. Methods of patient recruitment, image acquisition and analysis of MR imaging are described elsewhere in more detail $[22,37]$.

A group of 32 SPECT scans from non head injured patients (age mean $=44.94, \mathrm{SD}=16.78$ ), were used as a control group. These scans were acquired from archived data of patients referred to the neurological unit for investigation between 1993 and 1998 whose SPECT scans were found to be normal by experienced reporters and who had had no further history of neurological investigation.

Functional lesion sizes were obtained by using SPM'96 to compare each scan to a group of 32 normal scans with a replication of conditions experiment. The results were considered significant at a height threshold of $p<0.001$ and corrected extent threshold of $p<0.05$. Figure 2 shows an example of blood flow abnormalities identified by SPM in a head injured patient at the acute stage. Blood flow abnormalities are present in left temporal lobe and bilaterally in the medial frontal lobe. MR performed on the same occa- 

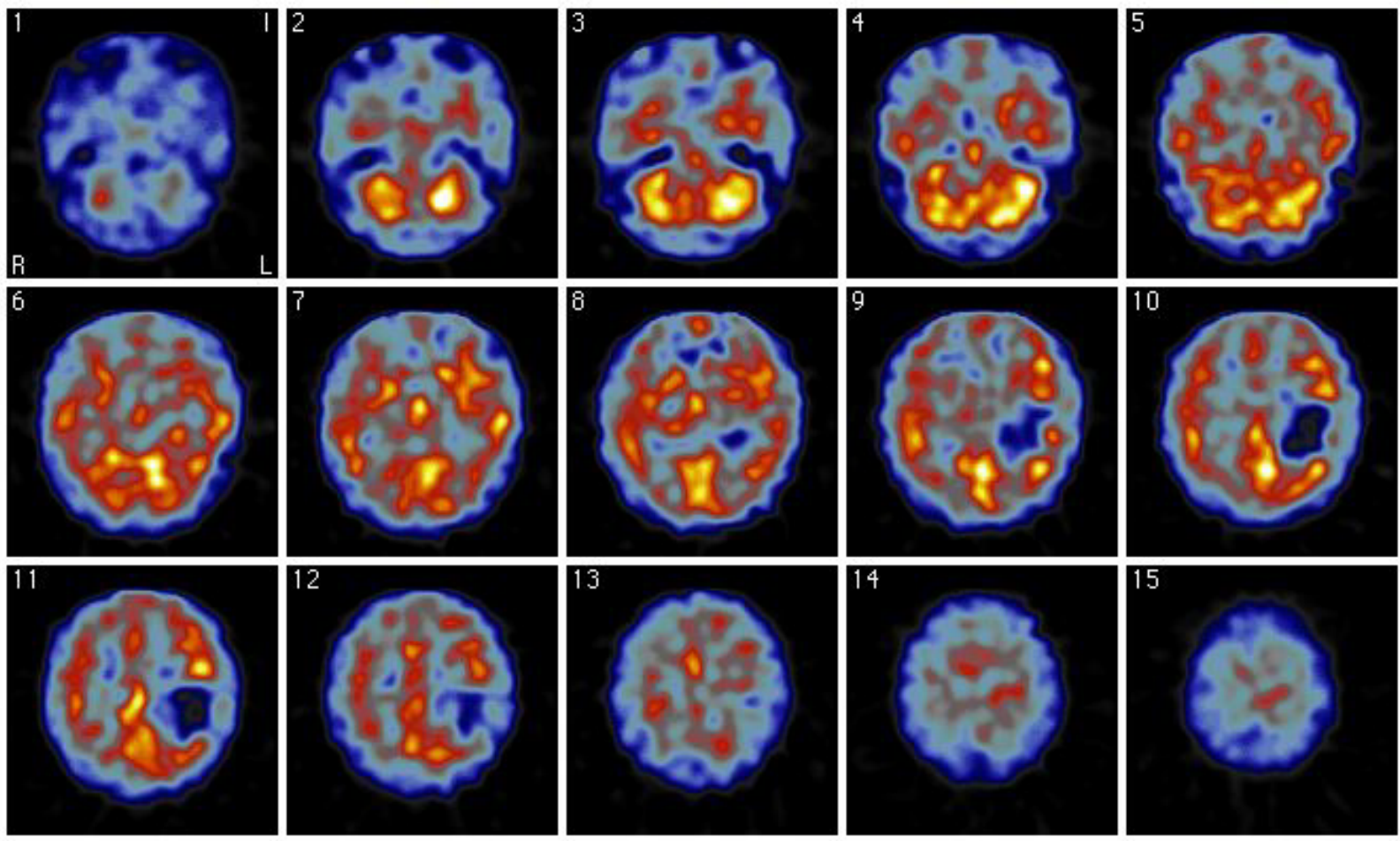

Fig. 3. SPECT full brain data set (slice $1=$ lowest slice, slice $15=$ highest slice) of a head injured patient corresponding to the analysis in 2 . An obvious focal abnormality can be seen in the left hemisphere and there is low blood flow in frontal areas (anterior regions are at the top of the slices).

sion revealed only lesions in the left hemisphere. The SPECT scan of the same patient can be seen in Fig. 3.

The total lesion volume for each patient's SPECT and MRI were computed (results for twenty patients are shown in Fig. 4). The results indicate that in the cases examined, SPECT detects in total more lesion volume than MRI, even though SPM underestimates the volume of lesions with small deviation from normal flow (Fig. 1). It is possible to have normal perfusion in MRI abnormal regions but this is rare. The study shows that there are differences between the volume of structural lesions measured with MRI and functional lesions measured by SPECT. Anatomically healthy tissue as indicated by MRI does not guarantee normal function especially in cases of diffuse head injury. This is due in part to axonal damage affecting innervation of MRI normal tissue [38].

\subsection{Neuropsychological test performance and blood flow deficits}

It is usually assumed that brain lesions after head injury are related to eventual cognitive deficit. However, it has proven difficult to demonstrate relationships between CT and MR lesions in specific locations and deficits on particular cognitive tests after head injury. Goldenberg and Oder [10,27] have investigated the relationship between cognitive problems after trauma and HMPAO SPECT. Goldenberg et al. [10] administered a neuropsychological battery which emphasised memory and executive functions but did not find the expected relationships between memory and temporal blood flow and between executive functions and frontal blood flow. They found general relationships between test performance and blood flow in frontal and thalamic areas. Oder et al. [27] investigated behavioural and psychosocial problems in the same patient group. The results supported the idea that disinhibition syndrome after head injury is due to orbito-frontal damage. Both studies $[10,27]$ suggest that there is a stronger relationship between personality change and blood flow than between cognitive deficit and blood flow. Wiedmann et al. [37] investigated relationships between blood flow deficits on HMPAO SPECT and neuropsychological test performance. The group of patients with focal injuries presented few significant associations most probably because this group showed little neuropsychological impairment. However, in the diffuse group nine of 


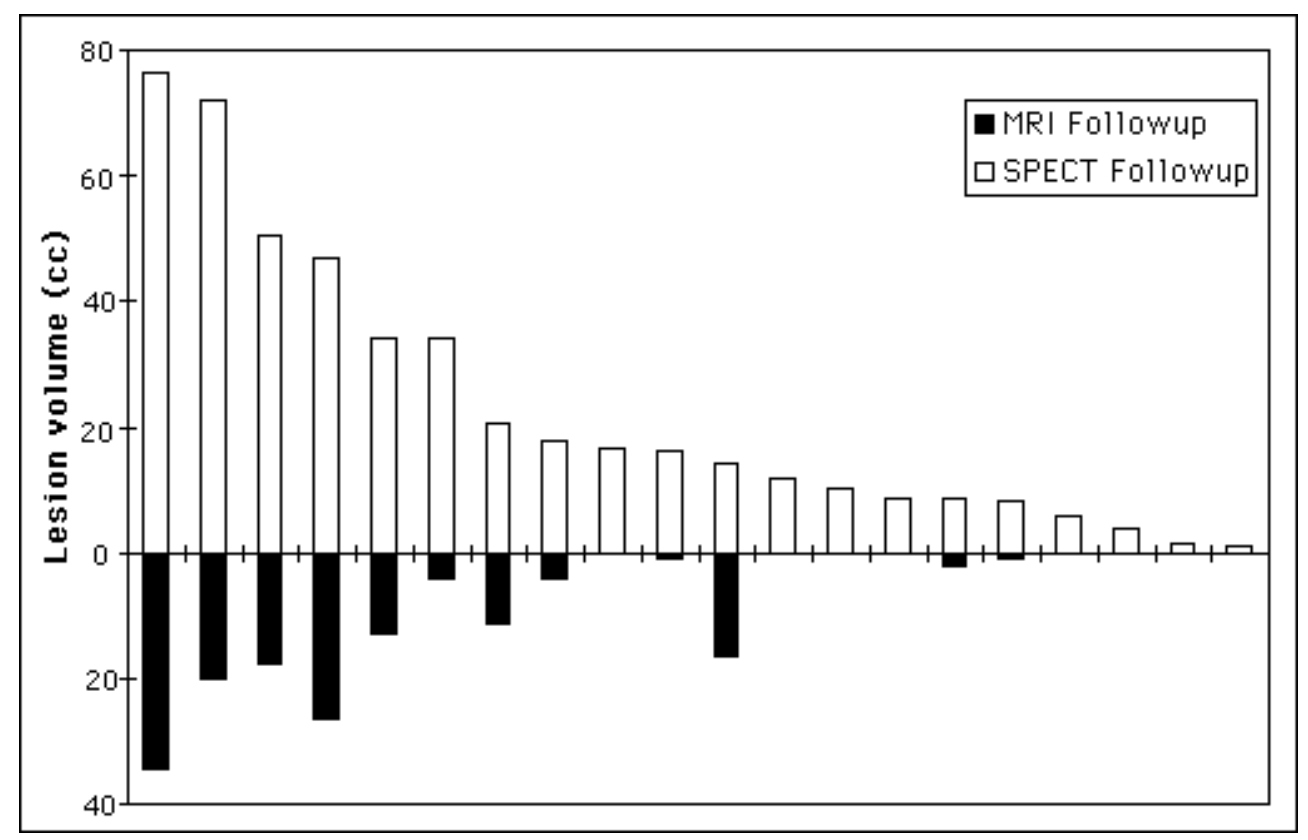

Fig. 4. Differences between total MRI lesion volume and total SPECT lesion volume in 20 head injured patients.

Table 1

Pearson correlation coefficients between the Rey Figure Immediate Recall test scores and various measures of overall lesion size from SPECT and MR; the correlation coefficient with age is also provided

\begin{tabular}{lc}
\hline & Rey Figure Test \\
\hline SPECT Acute & -0.135 \\
SPECT Follow - up & -0.166 \\
\% SPECT size change & 0.084 \\
MRI Acute & 0.054 \\
MRI Follow-up & 0.034 \\
\% MRI size change & -0.022 \\
Age & -0.146 \\
\hline
\end{tabular}

the 12 neuropsychological tests utilised were related to blood flow abnormalities. SPECT blood flow imaging is a promising technique for relating abnormalities to cognitive deficits [36], but the lack of sensitive methods of analysis has been a significant barrier to progress.

The application of SPM to this issue is illustrated here for a test of visual memory: the Rey Figure Immediate Recall. This test is part of a battery of tests completed by head injured patients on the same occasion as follow-up SPECT imaging. The Rey Figure is a widely used test of long-term visual memory that is known to be sensitive to the effects of head injury. It is commonly believed that frontal lobe lesions after head injury typically cause poor performance on the Rey Figure test [21]. However, there is little direct evidence for this view.

Brain damage after head injury is usually diffuse, and methods of analysis of brain imaging data often use global measures. Correlation coefficients between Rey Figure Immediate Recall test performance in 62 patients and total SPECT and MR lesion volumes are shown in Table 1. This analysis takes no account of the location of lesions and none of the relationships are statistically significant. This type of measurement might be more appropriate in the acute management of patients where it could be used along with intracranial pressure, arterial blood pressure, core temperature, oxygen saturation and heart rate and may improve the prognostic and patient management power of monitoring systems [29].

The effect of lesion location can be studied by using SPM to compare test performance to localised blood flow variation in the head injured patients. A covariate only design was employed with the test score as a covariate of interest and age a confounding covariate. Unwanted age effects were thus removed. The results were obtained at a height threshold of $p<0.001$ and corrected extent threshold of $p<0.05$. For this type of experimental design SPM produces results that are very similar in appearance to the results obtained from lesion detection experiments and include SPM maps superimposed on the glass brain (Fig. 5), and lists of local maxima with their Talairach coordinates and $\mathrm{Z}$ scores. SPM allows the user to superimpose the maps on a T1 image to assist the visualisation of corresponding anatomical structures (see Fig. 6). The figure demonstrates areas in which positive correlation was 

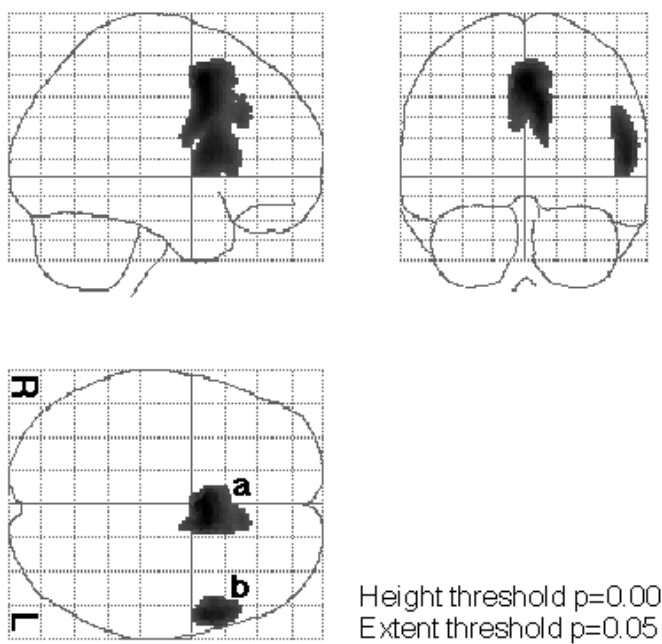

Height threshold $p=0.001$

Extent threshold $\mathrm{p}=0.05$ corrected

Fig. 5. Positive correlations between performance on the Rey Figure immediate recall test and blood flow. Maxima for the clusters shown here are at a) $2,10,42(Z=4.55)$ in the right cingulate (BA 32) and b) $-55,12,12(Z=4.05)$ in the left inferior frontal gyrus (BA 44).
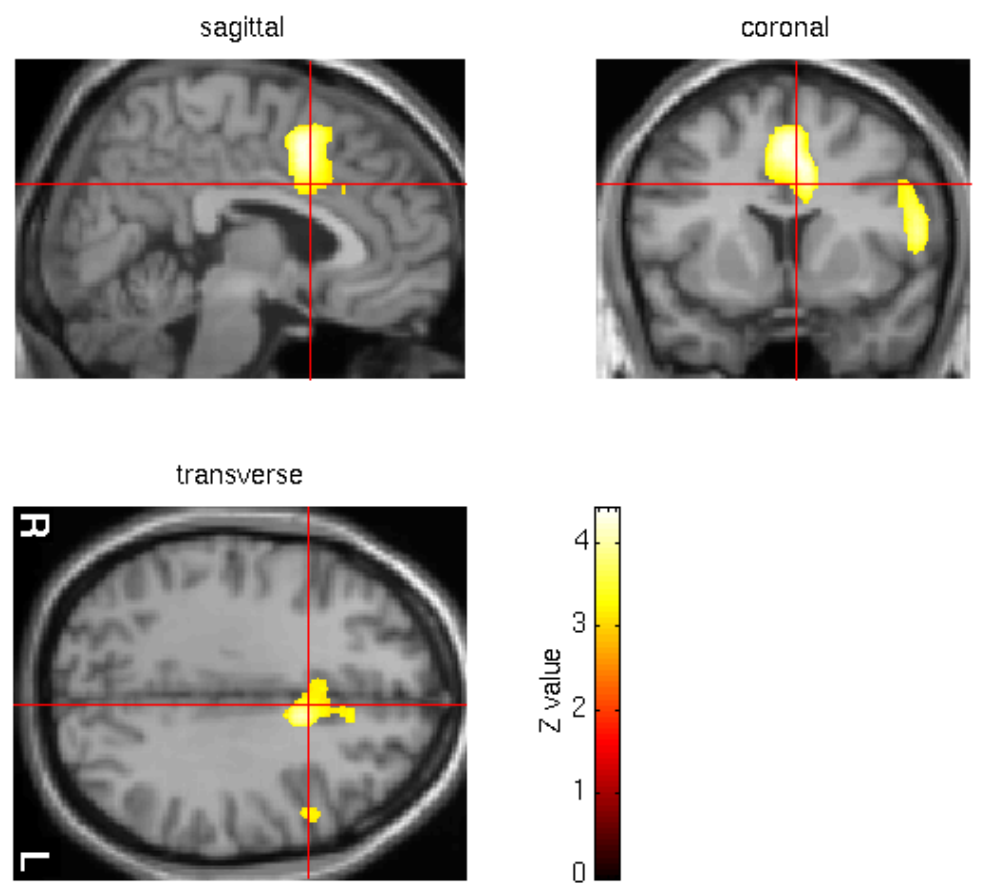

Fig. 6. Positive correlations between performance on the Rey Figure immediate recall test and blood flow (as in Fig. 5) superimposed on a T1 image. The red cross hair indicates transverse, sagittal and coronal planes.

achieved with neuropsychological scoring for the Rey Figure Immediate Recall test. Local maxima indicate voxels with maximum correlation. When all the areas on the SPM maps were considered we found that performance on the Rey Figure Test was related to blood flow in the medial frontal region (including cingulate 
cortex), and left frontal cortex (Fig. 6).

These preliminary findings suggest that SPM can be used to relate blood flow variation in localised regions to cognitive test performance after head injury. The specific results support the view that impaired Rey Figure performance after head injury is related to frontal lobe dysfunction [21]. Such demonstrations are potentially important because they provide direct evidence that cognitive test performance is related to brain lesions after head injury, and thus support the clinical interpretation of neuropsychological assessment. This type of analysis may also help to identify regions of the brain that are dysfunctional after head injury, and could potentially contribute to understanding the localisation of cognitive functions.

\section{Conclusion}

The results from the synthetic lesion studies show that SPM'96 can efficiently identify perfusion deficits on SPECT scans. The method established to analyse SPECT scans using SPM is an important step in the effort to quantify SPECT interpretation. Attempts to relate cognitive impairment after head injury to SPECT are often based on global measures of blood flow deficit and the results have generally been disappointing. The problem can be addressed with the use of SPM which produces location specific results by examining single voxels. The results suggest that SPM may have an important role to play in SPECT lesion studies by relating cognitive functions to specific brain areas.

\section{References}

[1] H.M. Abdel-Dayem, S.A. Sadek, K. Kouris, R.H. Bahar, I. Higazi, S. Eriksson, S.H. Engelesson, L. Berntman, G.H. Sigurdsson, M. Foad and H. Olivecrona, Changes in Cerebral perfusion after acute head injury: Comparison of CT with Tc-99m HMPAO SPECT, Radiology 165 (1987), 221-226.

[2] P.D. Acton and K.J. Friston, Statistical parametric mapping in functional neuroimaging: beyond PET and fMRI activation studies, European Journal of Nuclear Medicine 25 (1998), 663-667.

[3] P. Bartenstein, S. Minoshima, C. Hirsch and K. Buch et al., Quantitative assessment of cerebral blood flow in patients with Alzheimer's disease by SPECT, Journal Of Nuclear Medicine 38 (1997), 1095-1101.

[4] S. Bavetta, C.C. Nimmon, J. White, J. McCabe, A.H. Huneidi, J. Bomanji, B. Birkenfeld, M. Charlersworth, K.E. Britton and R.J. Greenwood, A prospective study comparing SPECT with MRI and CT as prognostic indicators following severe closed head injury, Nuclear Medicine Communications 15 (1994), 961-968.
[5] R. Bullock, P. Stathman, J. Patterson, D. Wyper, D. Hadley and E. Teasdale, The time course of vasogenic oedema after focal head injury - Evidence from SPECT mapping of blood brain barrier defects, Acta Neurochirurgica 51 (1990), 286-288.

[6] M.S. Choksey, D.C. Costa, F. Iannotti, P.J. Ell and H.A. Crockard, 99TCm-HMPAO SPECT studies in traumatic intracerebral haematoma, Journal of Neurology, Neurosurgery and Psychiatry 54 (1991), 6-11.

[7] D.C. Costa, P.J. Ell, A. Burns, M. Philpot and R. Levy, CBF tomograms with $99 \mathrm{~m}$ Tc-HM-PAO in patients with dementia (Alzheimer type and HIV) and Parkinson's disease - initial results, Journal of Cerebral Blood Flow Metabolism 8 (1988), S109-S115.

[8] K.J. Friston, Statistical parametric mapping, Functional Neuroimaging, R.W. Thatcher, M.Hallet, T.Zeffiro, E.R. John and M. Huerta, Eds., Academic Press, New York, 1994, pp. 79-93.

[9] K.J. Friston, A.P. Holmes, K.J. Worsley, J.P. Poline, C.D. Frith and R.S.J. Frackowiak, Statistical parametric maps in functional imaging: a general linear approach, Human Brain Mapping 2 (1995), 189-210.

[10] G. Goldenberg, W. Oder, J. Spatt and I. Podreka, Cerebral correlates of disturbed executive function and memory in survivors of severe closed head injury: a SPECT study, Journal of Neurology Neurosurgery \& Psychiatry 55 (1992), 362-368.

[11] B.G. Gray, M. Ichise, D.-G. Chung, J.C. Kirsh and W. Franks, Technetium-99m-HMPAO-SPECT in the evaluation of patients with a remote history of traumatic brain injury: A comparison with x-ray computed tomography, Journal of Nuclear Medicine 33 (1992), 52-58.

[12] A.S. Houston, P.M. Kemp and M.A. Macleod, A method for assessing the significance of abnormalities in HMPAO brain SPECT Images, Journal Of Nuclear Medicine 35 (1994), 239244.

[13] A.S. Houston, P.M. Kemp, M.A. Macleod, T. James, R. Francis, H.A. Colohan and H.P. Mathews, Use of significance image to determine patterns of cortical blood flow abnormality in pathological and at-risk groups, Journal of Nuclear Medicine 39 (1998), 425-430.

[14] M. Ichise, D.-G. Chung, P. Wang, G. Wortzman, B.G. Gray and W. Franks, Technetium-99m-HMPAO SPECT, CT and MRI in the evaluation of patients with chronic traumatic brain injury: A correlation with neurophychological performance, Journal of Nuclear Medicine 35 (1994), 217-226.

[15] A. Jacobs, E. Put, M. Ingels and A. Bossuyt, Prospective evaluation of Technetium-99m HMPAO SPECT in mild and moderate traumatic brain injury, Journal of Nuclear Medicine 35 (1994), 942-947.

[16] A. Jacobs, E. Put, M. Ingels, T. Put and A. Bossuyt, One-year follow-up of technetium-99m-HMPAO SPECT In mild headinjury, Journal Of Nuclear Medicine 37 (1996), 1605-1609.

[17] K.A. Johnson, M.F. Kijewski, J.A. Becker, B. Carada, A. Satlin and B.L. Holman, Quantitative brain SPECT in Alzheimer's Disease and normal ageing, Journal of Nuclear Medicine 34 (1993), 2044-2048.

[18] L.S. Johnson, R.S. Tikofsky, V. Furman, J.J. Burrascano, G.T. Simons, B.A. Fallon and R.L. VanHeertum, Reduced cerebral blood flow to white matter of Lyme Disease patients demonstrated by statistical parametric mapping of brain SPECT, Journal of Nuclear Medicine 40 (1999), 1997.

[19] H. Karbe, A. Kertesz, J. Davis, B.J. Kemp, F.S. Prato and R.L. Nicholson, Quantification of functional deficit in AlzheimersDisease using a computer-assisted mapping program for Tc99m-HMPAO SPECT, Neuroradiology 36 (1994), 1-6. 
[20] J.S. Lee, D.W. Lee, D.S. Lee, K.M. Kim, K.S. Park, J.K. Chung, M.J. Cho and M.C. Lee, Preserved cerebral perfusion reserve in Alzheimer's disease assessed by quantitative acetazolamide SPECT and statistical parametric mapping: Evidence against vascular factor hypothesis, Journal of Nuclear Medicine 40 (1999), 1221.

[21] M.D. Lezak, Neuropsychological assessment (3rd ed.), University Press, Oxford 1995

[22] A. Mitchener, D.J. Wyper, J. Patterson, D.M. Hadley, P. Mathew, J.T.L. Wilson, L.C. Scott, M. Jones and G.M. Teasdale, SPECT, CT and MR imaging in head injury: Acute abnormalities followed up at 6 months, Journal of Neurology, Neurosurgery, \& Psychiatry 62 (1997), 633-636.

[23] D. Montaldi, D.N. Brooks, J.H. McColl, D. Wyper, J. Patterson, E. Barron and J. McCulloch, Measurements of regional cerebral blood flow and cognitive performance in Alzheimer's disease, Journal of Neurology, Neurosurgery, and Psychiatry 53 (1990), 33-38.

[24] K.G. Nedd, G. Sfakianakis, W. Ganz, B. Uricchio, D. Vernberg, P. Villanueva, A.M. Jabir, J. Bartlett and J. Keena, 99m Tc-HMPAO SPECT of the brain in mild to moderate traumatic brain injury patients: compared with CT- a prospective study, Brain Injury 7 (1993), 469-479.

[25] M.R. Newton, R.J. Greenwood, K.E. Britton, M. Charlesworth, C.C. Nimmon, M.J. Carroll and G. Dolke, A study comparing SPECT with CT and MRI after closed head injury, Journal of Neurology, Neurosurgery, \& Psychiatry 55 (1992), 92-94.

[26] W. Oder, G. Goldenberg, I. Podreka and L. Deecke, HMPAO-SPECT in persistent vegetative state after head injury: prognostic indicator of the likelihood of recovery? Intensive Care Medicine 17 (1991), 149-153.

[27] W. Oder, G. Goldenberg, J. Spatt, I. Podreka, H. Binder and L. Deecke, Behavioural and psychosocial sequelae of severe closed head injury and regional cerebral blood flow: A SPECT study, Journal of Neurology, Neurosurgery, \& Psychiatry 55 (1992), 475-480.

[28] G.D. Pearlson, G.J. Harris, R.E. Powers, P.E. Barta, E.E. Camargo, G.A. Chase, J.T. Noga and L.E. Tune, Quantitative changes in mesial temporal volume, regional cerebral blood flow, and cognition in Alzheimer's disease, Archives of Gen- eral Psychiatry 49 (1992), 402-408.

[29] I. Piper and W. Payne, Adding value to monitored data, International Journal of Intensive Care 5 (1998), 140-141.

[30] S.N. Roper, I. Mena, W.A. King, J. Schweitzer, K. Garrett, C.M. Mehringer and D. McBride, An analysis of cerebral blood flow in acute closed-head injury using technetium-99mHMPAO SPECT and computed tomography, Journal of $\mathrm{Nu}$ clear Medicine 32 (1991), 1684-1687.

[31] D.E. Sakas, M.R. Bullock, J. Patterson, D. Hadley, D.J. Wyper and G.M. Teasdale, Focal cerebral hyperemia after focal head injury in humans: a benign phenomenon? Journal of Neurosurgery 83 (1995), 277-284.

[32] E.A. Stamatakis, J.T.L. Wilson and D.J. Wyper, The effect of non-linear image registration on cerebral lesions, Proceedings of Medical Image Understanding and Analysis 99 (July 1999), 21-24, Oxford.

[33] E.A. Stamatakis, M.F. Glabus, D.J. Wyper, A. Barnes and J.T.L. Wilson, Validation of Statistical Parametric Mapping (SPM) in assessing Cerebral lesions: A simulation study, NeuroImage 10 (1999), 397-407.

[34] G.M.S. Syed, S. Eagger, B.K. Toone, R. Levy and J.J. Barrett, Quantification of regional cerebral blood flow ( $\mathrm{rCBF}$ ) using 99mTc-HMPAO and SPECT: choice of a reference region, Nuclear Medicine Communications 13 (1992), 811-816.

[35] R.S. Tikofsky, S.P. Jonas, D. Singh, V. Furman, J.C. Brust, J.L. Curtis, R.C. Locko and R.L.VanHeertum, Statistical parametric mapping (SPM) in the evaluation of dementia: A pilot study, Journal of Nuclear Medicine 40 (1999), 1205.

[36] K.D. Wiedmann, J.T.L. Wilson, D. Wyper, D.M. Hadley, G.M. Teasdale and D.N. Brooks, SPECT cerebral blood flow, MR Imaging and neuropsychological finding in traumatic head injury, Neuropsychology 3 (1989), 267-281.

[37] J.T.L. Wilson, D.M. Hadley, L.C. Scott and A. Harper, The neuropsychological significance of contusional lesions identified by MR imaging, in: Recovery after traumatic brain injury, B.P. Uzzell and H.H. Stonnington, eds., Lawrence Erlbaum, Hillsdale, NJ, 1996.

[38] J.T.L. Wilson and D. Wyper, Neuroimaging and neuropsychological functioning following closed head injury: CT MR, and SPECT, Journal of Head Trauma Rehabilitation 7 (1992), 29-39. 


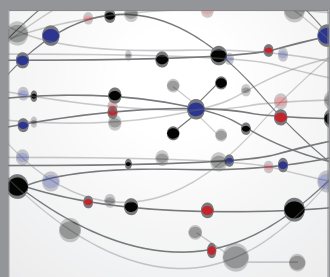

The Scientific World Journal
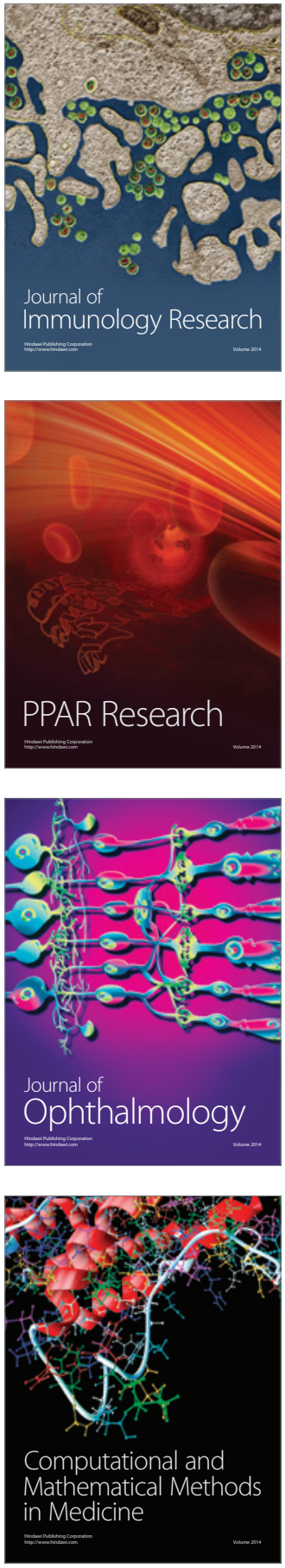

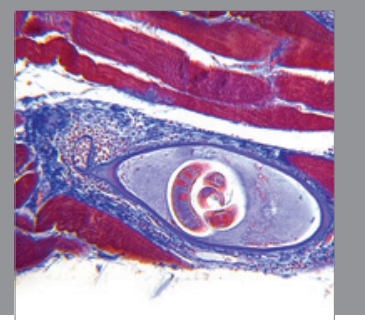

Gastroenterology

Research and Practice
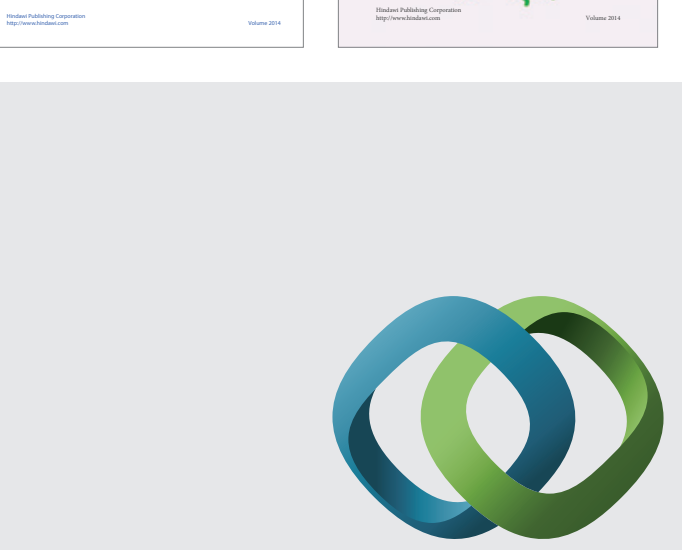

\section{Hindawi}

Submit your manuscripts at

http://www.hindawi.com
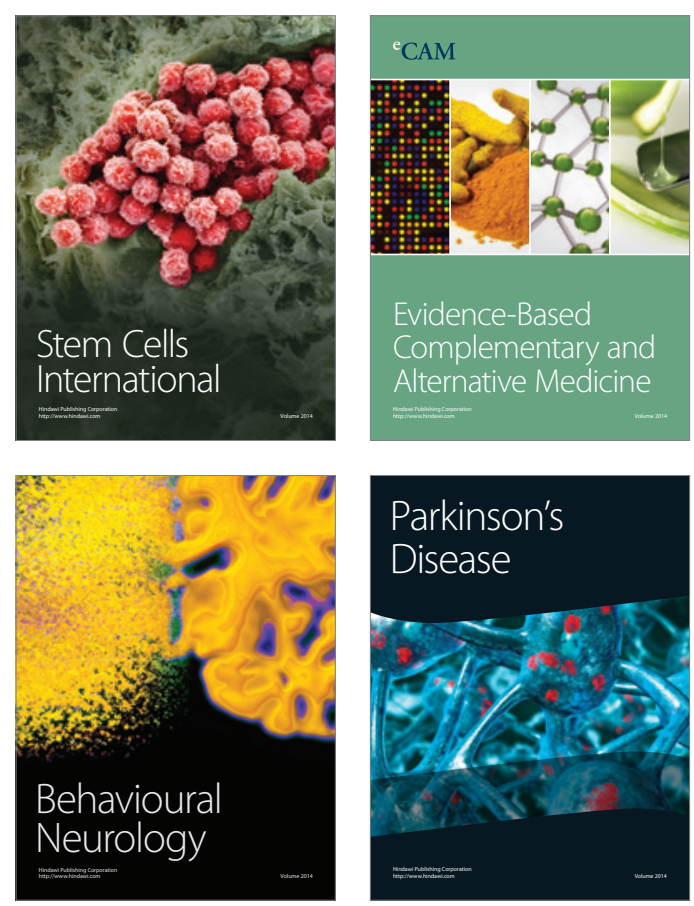

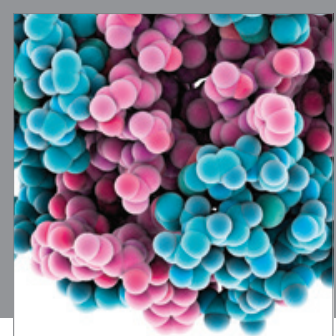

Journal of
Diabetes Research

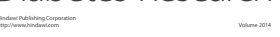

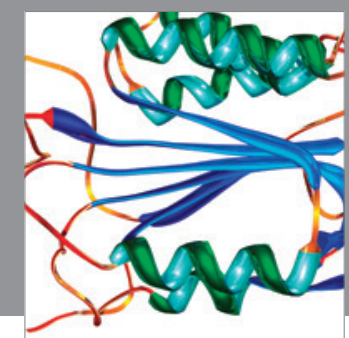

Disease Markers
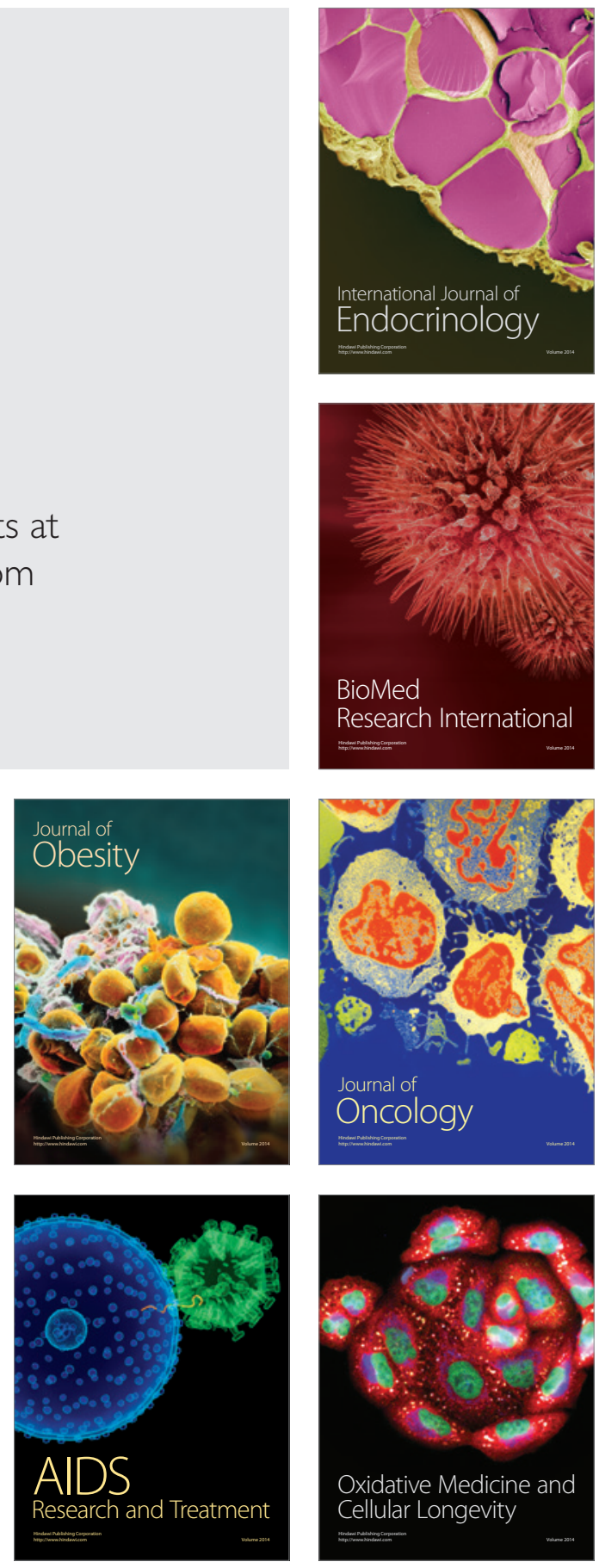